\title{
Comment on National Survey of Endoscopic Thymectomy: survey report from the Japanese Association for Research on the Thymus
}

\author{
Koji Kikuchi, MD
}

Received: 7 October 2008

(C) The Japanese Association for Thoracic Surgery 2009

Reports on the effectiveness of endoscopic thymectomy on diseases of the thymus such as myasthenia gravis or thymoma have been presented in recent years. Longterm postoperative care and observations have been included in those reports. In Japan, however, we have seen few reports presented about the analyses made to date.

The survey report from the Japanese Association for Research on the Thymus was based on answers to questionnaires sent to member facilities or to members of the Japanese Association for Research on the Thymus, aimed at determining the extent to which endoscopic thymectomy or how prevalent it is. The facilities and members who responded to the questionnaires were those who were performing endoscopic thymectomy. In view of these circumstances and from the nature of the cases, we cannot help feeling that this report is rather on the affirmative, "pro" side of the issue.

One of the problems of this survey report is that there are no references to complications of endoscopic thymectomy. We should, however, give great consideration to intraoperative complications, especially massive bleeding from the brachiocephalic vein. The report makes no reference to massive bleeding - how many cases experienced massive bleeding and needed intraoperative hemostasis with the help of median sternotomy.

This editorial refers to the article by Shiono et al. on pp. 22-27 of this issue of General Thoracic and Cardiovascular Surgery.

K. Kikuchi $(\bowtie)$

Department of General Thoracic Surgery, Saitama Medical Center, Saitama Medical University, 1981 Kamoda, Tsujidomachi, Kawagoe 350-8550, Japan

Tel. +81-49-228-3459; Fax +81-49-228-3459

e-mail: 28kkiku@saitama-med.ac.jp
There was no survey of such problems in the questionnaire. In 2005, during the 18th annual meeting of the Japan Society for Endoscopic Surgery, some reports were given on massive bleeding that occurred while endoscopic thymectomy was being performed. Among those, there was a report of a case that involved massive bleeding of more than $10000 \mathrm{ml}$. Taking the chair at the meeting, I saw that there were few facilities that reported cases of massive bleeding. The argument was that they had managed to cope with it and achieved a recovery with little difficulty, and therefore it was not a serious problem.

Nevertheless, when seen nationwide, despite the fact that there have been a number of cases of death due to massive bleeding from brachiocephalic vein injury, we have no information about such cases from the Japan Society for Endoscopic Surgery and the Japanese Association for Chest Surgery. Reports have been presented only from those facilities or members who experienced successful performances or results. This may be because those facilities where there have been patient deaths from such complications cannot willingly make public those facts, and they have avoided presenting those experiences at the conference meeting. This seems to be a very big problem.

The other problem is that the report makes no reference at all to long-term prognosis. At this time it seems a little too early to make such an evaluation. The effectiveness of endoscopic thymectomy for myasthenia gravis should be evaluated for at least 5 years, or more, for postoperative prognoses. A 5-year postoperative period is really too short; it will require 10 years to come up with the prognosis. Thus I cannot tell the effectiveness of endoscopic thymectomy on the basis of the questionnaire. 
Furthermore, since most items on the questionnaire seem to be from a rather affirmative point of view about endoscopic thymectomy, it would be premature to draw the conclusion, on the basis of that questionnaire, that endoscopic thymectomy has been recognized as an acceptable and prevailing method of treatment.

There was a symposium titled "Can endoscopic thymectomy be a standard operating method?" at the 27 th annual meeting of the Japanese Association for Research on the Thymus held in February 2008. There were presentations from 9 symposiasts from across Japan, followed by a discussion about the pros and cons of endoscopic thymectomy. Seven of the symposiasts asserted the effectiveness of endoscopic thymectomy; two of them stressed the significance of median sternotomy. After those presentations and discussions, Dr. Akira Masaoka and Dr. Takeshi Yoshitake commented. Both of them have played a leading role in surgical treatment of the thymus. In their commentary, they pointed out that "As yet, much remains unknown about surgical treatment of the thymus. Evaluation of endoscopic thymectomy without median sternotomy is still very difficult. We would rather you performed thymus surgery; confronting the problems of median sternotomy itself does not require so complicated and difficult a technique and does not take so much time and trouble."

These words from Dr. Masaoka and Dr. Yoshitake concluded the symposium on endoscopic thymectomy and treatment at the 27th annual meeting of the Japanese Association for Research on the Thymus. And with those words, I would like to conclude my comment on this survey report.

\section{Reference}

1. Shiono H, Okumura M, Yasumoto K. National Survey of Endoscopic Thymectomy: survey report from the Japanese Association for Research on the Thymus. Gen Thorac Cardiovasc Surg 2008;57:22-7. 